\section{Notifizierte Stelle}

U. Zimmermann ${ }^{1}$ und A. Steinhorst ${ }^{2}$

${ }^{1}$ Gesundheit / Forensik, Deutsche Akkreditierungsstelle

GmbH (DAkkS), Frankfurt, Deutschland

${ }^{2}$ EA Secretariat, Paris, Frankreich

Englischer Begriff notification; notified body

Definition Notifizierung: Mitteilung an andere Regierungen über die Benennung einer Konformitätsbewertungsstelle oder Akkreditierungsstelle.
Beschreibung Nach erfolgter Notifizierung kann eine benannte Stelle als notifizierte Stelle bezeichnet werden.

Benennung (von > Konformitätsbewertungsstelle): Staatliche Bevollmächtigung einer Konformitätsbewertungsstelle oder Akkreditierungsstelle, um festgelegte Konformitätsbewertungstätigkeiten unter Gesetzen oder Vorschriften durchzuführen.

\section{Literatur}

DIN EN ISO/IEC 17000:2005 „Konformitätsbewertung - Begriffe und allgemeine Grundlagen“" 